\title{
Outcomes after unilateral abscess tonsillectomy
}

\author{
Schrom T*, Tschuschke $O$ and Diederich LM \\ Department of Oto-Rhino-Laryngology, Helios Clinics Bad Saarow, Teaching hospital of the Brandenburg Medical School Theodor Fontane, Germany
}

\begin{abstract}
Background: To treat peritonsillar abscesses, needle puncture, incisional drainage, and abscess tonsillectomy (tonsillectomy à chaud) have been shown to be effective. In Germany, new guidelines were published in 2015 that recommend restricting simultaneous tonsillectomy of the contralateral side for only specific indications. To date, there have not been international studies to assess the clinical course or the quality of life after unilateral tonsillectomy (TE). The aim of this study was to assess the postoperative health-related quality of life and the course of the disease in patients that underwent surgical removal of only the affected palatinal tonsil in the setting of abscess formation.

Methods: Questionnaires were sent to 77 patients treated at Helios Klinikum Bad Saarow by unilateral tonsillectomy for abscess formation: the validated Glasgow Benefit Inventory (GBI) and the Specific Benefits from Tonsillectomy Inventory (SBTI). In addition, the clinical course of the patients was assessed based upon the charts in the hospital information system.

Results: 23 patients answered and returned the questionnaire (response rate 29.8\%). The mean age was 58 years (range: $26-78$ years). Seventy-four percent were male, $26 \%$ female. The results of the GBI showed no improvement in postoperative quality of life and the general health, and no improvement in social support and the physical functioning. In contrast, in the SBTI patients judge their symptoms after surgery as significantly improved and report a reduced use of resources such as antibiotics or doctor's visits, which was statistically significant (both $\mathrm{p}=0.002$ ). The overall improvement in quality of life is also reported to be only small. During a mean follow-up of 44 months, no patients had a PTA on the contralateral side and only 2 patients had post-operative hemorrhage (8.7\%), which stopped without intervention. Contralateral inflammation occurred more often in 5\%, at the same rate in $29 \%$, more rarely in $24 \%$ and much more rarely in $38 \%$.
\end{abstract}

Conclusion: The GBI as questionnaire on change in health-related quality of life shows no improvement post-surgery vs. pre-surgery. The SBTI as a tonsillitisspecific questionnaire showed significant improvements in symptoms and the use of resources compared to pre-surgery. The lack of contralateral abscess formation during follow-up physical functioning supports the advantage of unilateral abscess tonsillectomy.

\section{Introduction/background}

The palatinal tonsils (tonsillae palatinae) are part of Waldeyer's tonsillar ring. Peritonsillar and paratonsillar abscesses (PTA) can occur as a complication of acute bacterial tonsillitis or as an exacerbation of chronic tonsillitis. As possible causes of PTA are considered an infection proceeding from the tonsillar crypts, an abscess-forming infection of the His-duct, and an abscess formation of Weber's glands at the upper pole of the tonsil [1]. Often there is aerobic/anaerobic mixed infection with PTA.

In 2016, the diagnosis „chronic diseases of the palatinal tonsils and the pharyngeal tonsils "was the third most common diagnosis in children up to 15 years admitted to the hospital in Germany [2]. A PTA was the main diagnosis in 16,006 inpatient cases [3]. Risk factors for PTA include smoking, poor oral hygiene and male sex [4]. Bilateral abscesses are rare; one retrospective analysis reports a frequency about $3.9 \%$ [5]. If left untreated, PTA can result in potentially life-threatening complications [6].

Effective treatments of PTA are needle puncture, incisional drainage, and abscess tonsillectomy (tonsillectomy à chaud) [7]. Until 2015, PTA in Germany was often treated by bilateral tonsillectomy, to prevent the occurrence of a PTA on the other side. This changed substantially in 2015 with the publication of the S2k guideline "Therapy of inflammatory diseases of the palatinal tonsils- tonsillitis", of the German Society for ear nose and throat, head and neck surgery. The guideline's authors stated that simultaneous tonsillectomy of the contralateral side should only be performed according to the recommendations for tonsillectomy for tonsillitis or if there is evidence for a bilateral peritonsillar abscess [8].

Little is known about the clinical course and quality of life measures after unilateral abscess tonsillectomy. If so indicated, unilateral abscess tonsillectomy has been performed in our clinic already for more than 10 years. In this study, we analyzed patient satisfaction including the postoperative quality of life as well as the clinical course in our patients in whom only the affected palatinal tonsil was removed for abscess formation (so-called „tonsillectomy à chaud“). For this purpose, data were collected retrospectively using a questionnaire.

\section{Methods}

The study was approved by the Ethics Committee of the State Chamber of Physicians of Brandenburg (S 16(a)/2016). Inclusion criteria were age $>15$ years and a unilateral abscess tonsillectomy for PTA. Exclusion criteria were: elective tonsillectomy for recurring acute tonsillitis (RAT) and tonsillar hyperplasia as well as tonsillectomy for suspected neoplasia. Seventy-seven patients who underwent unilateral tonsillectomy for abscess formation between 2009 and 2016 at Helios

${ }^{\star}$ Correspondenceto: Thomas Schrom, MD, Department of Oto-Rhino-Laryngology, Helios Clinics Bad Saarow, Teaching hospital of the Brandenburg Medical School Theodor Fontane, Germany, E-mail: thomas.schrom@helios-gesundheit.de

Received: February 26, 2019; Accepted: March 15, 2019; Published: March 19, 2019 
Klinikum Bad Saarow were eligible for the study, and these patients were sent questionnaires and asked to participate.

We used the validated Glasgow Benefit Inventory (GBI). This internationally accepted questionnaire was developed to assess the change of health-related quality of life produced by a surgical procedure; patients are questioned once post-intervention [9]. It is primarily used in surgical ENT [10]. The GBI consists of 18 questions: 12 questions on general health, 3 questions on the relationship with family and friends/social support, and 3 questions on physical functioning. One advantage of the GBI is that both improvements and deteriorations can be documented. As a second questionnaire, we used the nonvalidated "Specific benefits from tonsillectomy inventory" (SBTI) [11]. It measures the change in disease-specific symptoms and the healthrelated quality of life after tonsillectomy. Questions 1 through 3 refer to typical symptoms of recurring acute tonsillitis, questions 4 through 6 assess the use of resources (antibiotics and doctor's visits), questions 7 and 8 assess the quality of life. In addition, we asked three questions regarding the subsequent clinical course and the occurrence of postoperative complications and infections of the contralateral side after hospital discharge.

The GBI and the SBTI provides 5 answer options for every question that reflect the degree change in symptoms. Both questionnaires were analyzed as follows: addition of the points (1-5) of every question and division by the number of questions. From the sum 3 is subtracted and then the result is multiplied with 50. Thus, possible values can range from "- 100" (maximal deterioration) to " +100 " (maximal improvement). The Wilcoxon-signed-rank test was used to test whether the mean of the scores was significantly different from zero (=no change). Statistical significance was accepted at $p<0.05$. Data are presented as mean \pm standard deviation unless stated otherwise.

In addition, the clinical course of the patients was assessed using the charts in the hospital information system. In all patient's cold steel tonsillectomy was performed under general anesthesia.

\section{Results}

Average follow-up was 44 months (3.6 years; range: 19-81 months). The average age of the 77 patients that were eligible for study inclusion was $46 \pm 18$ years (range $15-89$ years). The patients were $64 \%$ [ $n=49$ ] male and $36 \%[n=28]$ female. Forty-three percent [ $n=33]$ were smokers, $56 \%[n=43]$ non-smokers, one patient did not state his smoking status. The PTA was on the right in $47 \%[\mathrm{n}=36]$ and on the left in $53 \%$ [ $n=41]$. In our clinic the incidence of PTA across the year was uneven with a peak in May with almost double the number of patients (Figure 1). Mean duration of surgery was $22 \pm 8 \mathrm{~min}$ (range: 8-62 min). The average length of hospital stay was 6 days (range: 4-14 days). Pain intensity was assessed using the visual analog scale throughout the hospital stay (Figure 2). For analgesia, non-steroidal anti-inflammatory drugs were used.

Twenty-three of the 77 eligible patients answered and returned the questionnaire (response rate 29.8\%). The average age of responders was 58 years (range: $26-78$ years). Seventy-four percent were male, $26 \%$ female. The non-responding patients tended to be younger (average age 41 years; range: $15-89$ years); $59 \%$ were male and $41 \%$ female. However, the differences in age (Chi-square test: $p=0.34$ ) and gender distribution (Fisher's exact test $\mathrm{p}=0.17$ ) were not significant.

The GBI examines the postoperative change regarding the quality of life. The total GBI score (Figure 3 and Table 1) of all 23 patients showed no improvement in health-related quality of life with a mean of $2 \pm 20$ (range: -67 to +33 ). The mean sub score for general health was $2 \pm 23$

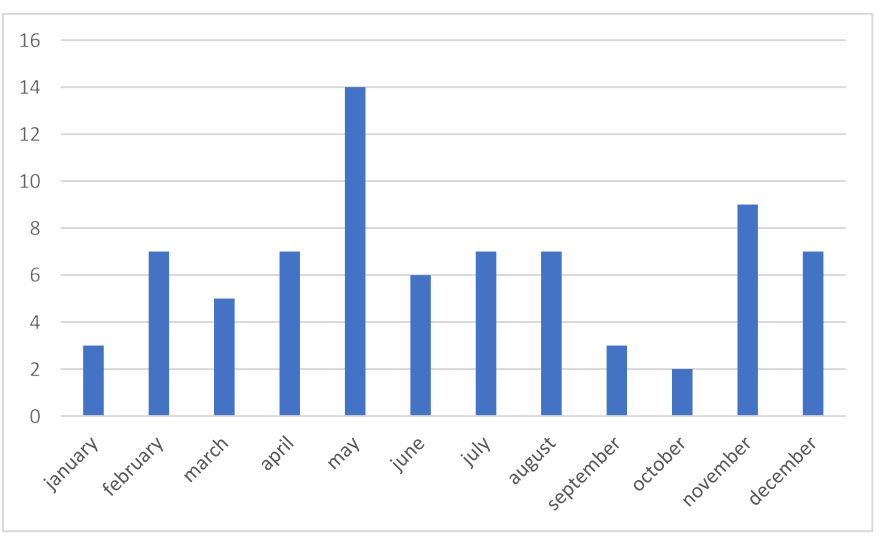

Figure 1. Cases of PTA per months (absolute numbers)

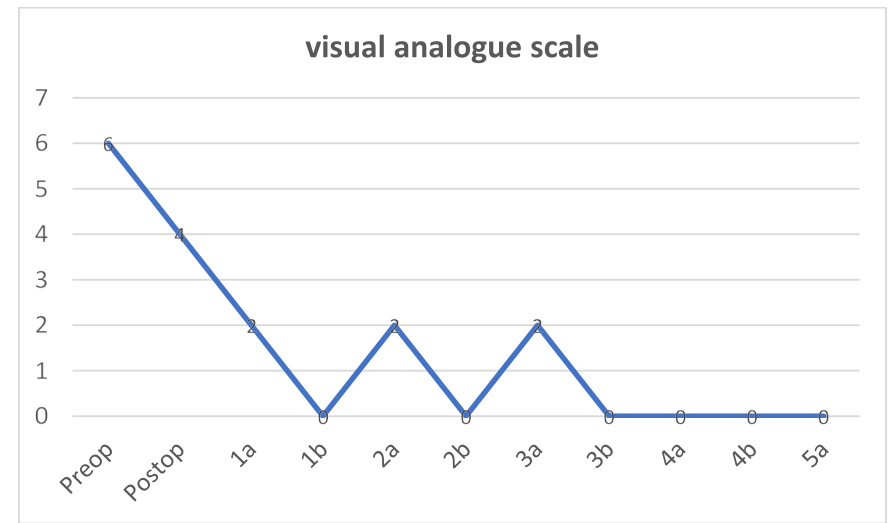

Figure 2. Pain intensity as assessed by visual analog scale (VAS) postoperatively after unilateral abscess tonsillectomy. Preoperatively: with abscess. Postoperatively: first assessment on the ward immediately after surgery. 1a: first post-operative day in the morning. 1b: first post-operative day in the afternoon. 2a: second post-operative day in the morning. 2b: second post-operative day in the afternoon. 3a: third post-operative day in the morning. 3b: third post-operative day in the afternoon. Etc

Table 1. Results of the GBI (Glascow Benefit Inventory) with mean and standard deviation of the overall score and subscores

\begin{tabular}{|c|c|c|}
\hline GBI & mean \pm SD & p-value \\
\hline Total score & $1,5 \pm 20$ & 0,229 \\
\hline Subscores & & \\
\hline General health & $1,5 \pm 23$ & 0,372 \\
\hline Social support & $0,8 \pm 14$ & 1 \\
\hline Physical functioning & $0 \pm 30$ & 0,904 \\
\hline
\end{tabular}

(range: -75 to +50 ). The mean subscore for social support was $1 \pm 14$ (range: -17 to +50$)$ and the mean subscore for physical functioning was $0 \pm 30$ (range: -83 to +67 ). None of the GBI scores reached statistical significance.

The SBTI (Figure 4 and Table 2) showed a significant improvement in disease-specific symptoms post-surgery with a mean score of $42 \pm$ 47 (range: -33 to +100 ). The mean subscore for reduction in the use of resources was $45 \pm 49$ (range: -66 to +100 ) and was also statistically significant. The mean subscore for general benefit was $10 \pm 39$ (range: -75 to +50$)$ and not statistically significant.

With regard to the clinical course, 2 patients had post-operative hemorrhage (8.7\%), which stopped without intervention; both occurred on the second postoperative day. Contralateral inflammation occurred more often in $5 \%(n=1)$, at the same rate in $29 \%(n=6)$, more rarely in $24 \%(n=5)$ and much more rarely in $38 \%(n=8)$. 


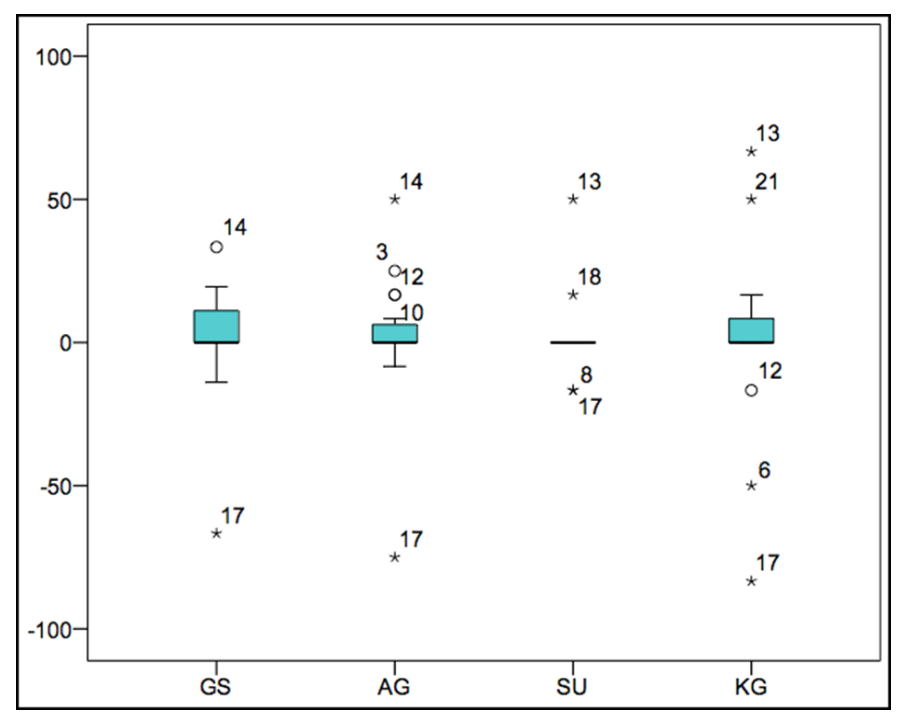

Figure 3. Results for the GBI: TS (total score), GH (general health), SS (social support), PF (physical functioning). Circle: outlier (1.5-3 times height of the box); star: extreme values ( $>3$ times the height of the box)

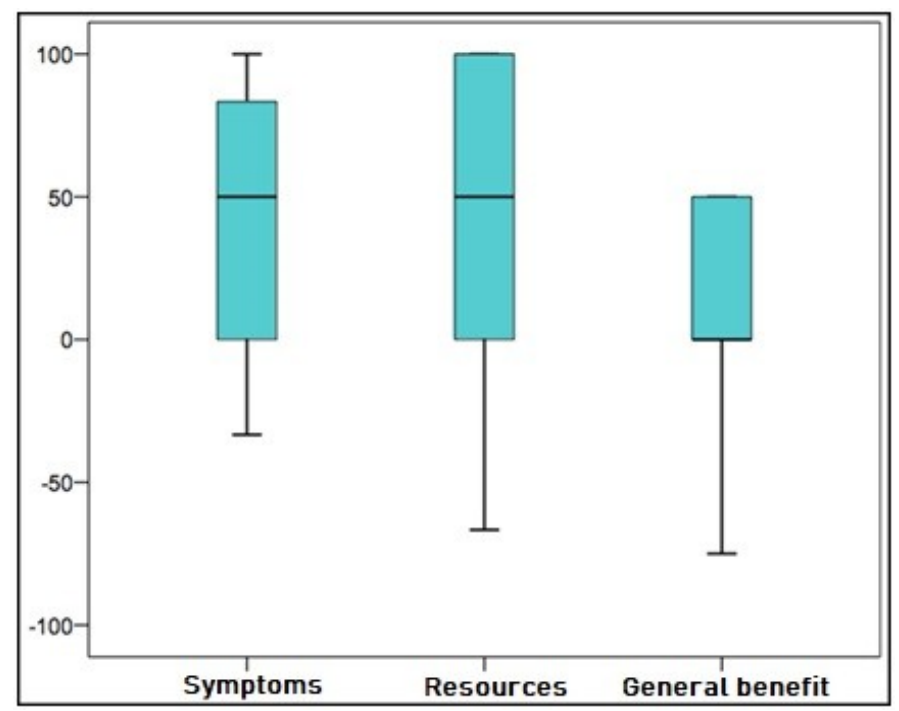

Figure 4. Results of the SBTI: Symptoms, resources and general benefit

Table 2. Results for the Specific Benefits from Tonsillectomy Inventory (SBTI) with mean and standard deviation

\begin{tabular}{|c|c|c|}
\hline SBTI & Mean \pm SD & p-value \\
\hline Symptom change & $41,7 \pm 47$ & 0,002 \\
\hline Reduced use of resources & $45,2 \pm 49$ & 0,002 \\
\hline General benefit & $10,2 \pm 39$ & 0,283 \\
\hline
\end{tabular}

\section{Discussion}

PTA is the most common complication of acute tonsillitis. In patients in whom there is no history of recurring contralateral acute tonsillitis, unilateral abscess tonsillectomy has already been the established therapy of choice in our clinic for years. Therefore, the mean follow-up is 3.6 years with a maximum of 81 months. In our study, during follow-up none of the patients had a PTA of the contralateral side and in only one there was an increase in inflammation of the contralateral side, so that, considering the surgical risk, our results support the advantage of leaving the contralateral side in situ. With regard to the patients' preoperative pain assessment per VAS, there was high pain intensity with a mean of 6 , which is caused by the PTA itself (Figure 2). Immediately post-surgery, pain is improved to a level of 4 , which is further reduced on the first postoperative day. Our patient group (mean age 58 years) was older compared to other studies that used the GBI to assess quality of life after elective tonsillectomy (e.g. 31.9 years in Baumann et al., 27.3 years in Bhattacharyya $\mathrm{N}$ et al., 28 years in Senska G et al., 26 years in Koskenkova et al.) [11-14]. The preponderance of men ( $74 \%$ male; $26 \%$ female) in our study is in contrast to the predominance of women in the GBI studies after elective tonsillectomy (e.g. 75.4\% women in Bhattacharyya $\mathrm{N}$ et al., $75 \%$ women in Senska G et al., 66\% women in Koskenkova et al.) [12,13,15]. This difference in gender distribution can be explained with male sex being a known risk factor for PTA [4].

The GBI is a validated questionnaire which was designed to assess predominantly the health-related quality of life after a surgical procedure in ENT. The boxplots of the GBI results show that individual answers varied widely between patients (Figure 4). Most patients rank their postoperative quality of life and their general health after unilateral abscess tonsillectomy as slightly improved. The social support as well as physical functioning are experienced as almost unchanged in comparison to preoperatively. Some patients report a deterioration, but none of the calculated overall scores or subscores is negative. Compared to reported GBI results after elective bilateral tonsillectomy in recurring acute tonsillitis and formerly chronic tonsillitis, respectively, patients with abscess tonsillectomy after PTA improve to a lesser degree in health-related quality of life. Bhattacharyya $\mathrm{N}$ et al., Senska G et al. and Baumann et al. reported an improvement of the total score $(+27.1 /+19 /+16.9)$, of the general health $(+34,7 /+18 /+12,9)$, of social support $(+14.4 / 0)$ and of physical functioning $(+9.5 /+39 /+46.6)$ in the GBI, which were all statistically significant [11-13]. These studies as well as our study report a tendency for a larger post-operative benefit in total score and subscore with regard to general health, as in the subscore social support by family/friends. Neither bilateral tonsillectomy nor unilateral abscess tonsillectomy appear to lead to a secondary morbid gain. The subscore physical functioning varied a lot in the studies. Koskenkorva et al. published 2009 and 2014 similar results of the GBI in patients after elective bilateral tonsillectomy. There was both an improvement in the total score $(+26 /+27)$, the general health $(+25)$, the social support by family and friends $(+5 / 0)$, and physical functioning $(+55 /+83)[14,15]$. In particular, the subscale physical functioning was assessed very positively as compared to the results of our study and the study by Bhattacharyya et al.

In contrast, in the SBTI patients in our study assessed symptoms after surgery as significantly better and reported a reduced use of resources such as antibiotic use, which was significant. Overall general benefit was only improved slightly and not significantly. Individual patients reported a worsening of symptoms in the SBTI. However, a definite change for the worse cannot be documented according to the scores. Baumann et al. used the SBTI to assess the benefit of elective bilateral tonsillectomy in chronic tonsillitis [11]. Compared to our study, the results of the subscores symptom change (Baumann: +58.3 / Schrom: +41.7 ), reduction in the use of resources (Baumann: +70.9 / Schrom: +45.2) and general benefit (Baumann: +52.1/Schrom: +10.2 ) showed positive changes across all 3 scores in both studies; of note, however, all subscores were consistently higher after elective tonsillectomy. Furthermore, several studies demonstrated a significant postoperative reduction in the number of sick days, doctor's visits and drug use (analgesics and antibiotics) $[12,13,16,17]$, which is comparable to our results of the SBTI. Despite significant symptom reduction and decreased use of resources, our study did not demonstrate a significant 
improvement in the quality of life. A possible explanation may be a limited number of preoperative infectious episodes in the setting of an acute tonsillitis.

The overall better assessment of the postoperative quality of life in the SBTI as compared to the GBI was also reported in another study [11]. The GBI is a more general questionnaire, which assesses the change in health status by an intervention, whereas the SBTI specifically captures the symptoms of tonsillitis. As with our study, in the available literature no change in the social support with elective tonsillectomy has been demonstrated; therefore, secondary gain from the disease does not play a major role [11].

Our study's response rate of $29.8 \%$ is low but comparable to similar studies (26.3\% Battacharyya [12]). The relatively long follow-up (44 months) may impact the patients' memory regarding the preoperative state (antibiotic usage, frequency of doctor's visits, etc.). However, the study by Schwentner et al. did not show a difference in GBI evaluation based on different lengths of questioning intervals [18]. The number of patients with post-operative hemorrhage $(n=2 ; 8.7 \%)$ is similar to what has been reported in the literature. The percentages range between $1.8 \%$ at the lower end and a higher rate of $13 \%$ with abscess tonsillectomies $[19,20]$. However, in both studies bilateral „tonsillectomy à chaud“ was performed in which the rate of post-operative hemorrhage was higher on the contralateral side. Therefore, a direct comparison with our rate of post-surgical hemorrhage is difficult.

\section{Conclusion}

Given the lack of PTA of the contralateral side during a follow-up of 44 months, our study supports performing unilateral abscess tonsillectomy when the current S2k guidelines „Therapy of inflammatory diseases of the palatal tonsils - tonsillitis" are taken into account. The GBI, as a more general questionnaire for change in healthrelated quality of life, showed that in unilateral abscess tonsillectomy, postoperatively there was only a slight improvement compared to preoperatively. The SBTI, a tonsillitis-specific questionnaire, showed a significant improvement compared to preoperatively; specifically, reduced symptoms and reduced use of resources.

\section{References}

1. Powell E, Powell J, Samuel J, Wilson J (2013) A review of the pathogenesis of adult peritonsillar abscess: time for a re-evaluation. J Antimicrob Chemother 68: 1941-1950.

2. Statistisches Bundesamt (2016) Vollstationar behandelte Kinder im Alter von 0 bis unter 15 Jahren in Kranken־hausern nach der ICD-10 im Jahr 2016. https://www. destatis.de/DE/ZahlenFakten/GesellschaftStaat/Gesundheit/Krankenhaeuser/Tabellen/ Diagnosen_Kinder.html
3. Statistisches Bundesamt (2016) Diagnosedaten der Patienten und Patientinnen in Krankenhausern (einschl. Sterbe- und Stundenfälle) 2016. https://www. destatis.de/DE/Publikationen/Thematisch/Gesundheit/Krankenhaeuser/ DiagnosedatenKrankenhaus2120621167004.pdf?_blob=publicationFile

4. Lehnerdt G, Senska K, Fischer M, Jahnke K (2005) Rauchen prädisponiert zum Peritonsillarabszess. Laryngo- rhino- otologie 84: 676-679.

5. Lehnerdt G, Senska K, Fischer M, Jahnke K (2005) Bilateral peritonsillar abscesses. Eur Arch Otorhinolaryngol 262: 573-575. [Crossref]

6. Federspil P (2009) Therapy options for peritonsillar abscess. HNO 57: 223-229. [Crossref]

7. Johnson RF, Stewart MG (2005) The contemporary approach to diagnosis and management of peritonsillar abscess. Current opinion in otolaryngology \& head and neck surgery 13: 157-160.

8. Berner R, Steffen G, Toepfer N, Waldfahrer F, Windfuhr J (2015) Therapie entzündlicher Erkrankungen der Gaumenmandeln - Tonsillitis. AWMF-Register Nr. 017/024.

9. Robinson K, Gatehouse S, Browning GG (1996) Measuring patient benefit from otorhinolaryngological surgery and therapy. Ann Otol Rhinol Laryngol 105: 415-422. [Crossref]

10. Hendry J, Chin A, Swan IRC, Akeroyd MA, Browning GG, et al. (2016) The Glasgow Bene?t Inventory: a systematic review of the use and value of an otorhinolaryngological generic patient-recorded outcome measure. Clin Otolaryngol 41: 259-275

11. Baumann I, Kucheida H, Blumenstock G, Zalaman IM, Maassen MM, et al. (2006) Benefit from tonsillectomy in adult patients with chronic tonsillitis. Eur Arch Otorhinolaryngol 263: 556-559. [Crossref]

12. Bhattacharyya N, Kepnes LJ, Shapiro J (2001) Efficacy and quality-of-life impact of adult tonsillectomy. Arch Otolaryngol Head Neck Surg 127: 1347-1350. [Crossref]

13. Senska G, Ellermann S, Ernst S, Lax H, Dost P (2010) Recurrent Tonsillitis in adults: quality of life after tonsillectomy. Dtsch Arztebl Int 107: 622-628.

14. Koskenkorva T, Koivunen P, Penna T, Teppo H, Alho OP (2009) Factors affecting quality-of-life impact of adult tonsillectomy. J Laryngol Otol 123: 1010-1014. [Crossref]

15. Koskenkorva T, Koivunen P, Laara E, Alho OP (2014) Predictive factors for quality of life after tonsillectomy among adults with recurrent pharyngitis: a prospective cohort study. Clin. Otolaryn-gol 39: 216-223.

16. Mui S, Rasgon BM, Hilsinger RL Jr (1998) Efficacy of tonsillectomy for recurrent throat infection in adults. Laryngoscope 108: 1325-1328. [Crossref]

17. Hsu AP, Tan KL, Tan YB, Han HJ, Lu PK (2007) Benefits and efficacy of tonsillectomy for recurrent tonsillitis in adults. Acta Otolaryngol 127: 62-64. [Crossref]

18. Schwentner I, Hofer S, Schmutzhard J, Deibl M, Sprinzl GM, et al. (2007) Impact of tonsillectomy on quality of life in adults with chronic tonsillitis. Swiss Med Wkly 137: 454-461. [Crossref]

19. Windfuhr JP, Remmert S (2005) [Trends and complications in the management of peritonsillar abscess with emphasis on children]. HNO 53: 46-57. [Crossref]

20. Giger R, Landis BN, Dulguerov P (2005) Hemorrhage risk after quinsy tonsillectomy. Otolaryngol Head Neck Surg 133: 729-734.

Copyright: (C2019 Schrom T. This is an open-access article distributed under the terms of the Creative Commons Attribution License, which permits unrestricted use, distribution, and reproduction in any medium, provided the original author and source are credited. 